\title{
MULTIPLE MELLIN-BARNES INTEGRALS AS PERIODS OF CALABI-YAU MANIFOLDS WITH SEVERAL MODULI
}

\author{
M.Passare, A.K.Tsikh, A.A.Cheshel
}

\begin{abstract}
We give a representation, in terms of iterated Mellin-Barnes integrals, of periods on multi-moduli Calabi-Yau manifolds arising in superstring theory. Using this representation and the theory of multidimensional residues, we present a method for analytic continuation of the fundamental period in the form of Horn series.
\end{abstract}

Superstring vacua with $(2,2)$ worldsheet supersymmetry, and also Landau-Ginzburg vacua, are determined by the special geometry of the moduli spaces of CalabiYau manifolds and of the corresponding orbifolds. Moreover, the deformation moduli of the complex structure and of the Kähler metric constitute two sectors in the moduli space, and they are in a $1-1$ correspondence with the 27 and $\overline{27}$ multibraids of the matter fields, which are $E_{6}$-charged in the low energy limit of the theory.

Thanks to the mirror symmetry for Calabi-Yau manifolds the sector of deformation moduli of the Kähler metric is identified with the sector of deformation moduli of the complex structure on the corresponding mirror manifold [1]. The dynamics of the matter fields in the low energy theory depends on the geometry of the moduli space, and both the kinetic term and the Yukawa coupling constants are determined by a holomophic object on the manifold $M$. This object is a holomorphic $(3,0)$-form $\Omega$, or more precisely the period vector of $\Omega$, having as components

$$
\varpi_{j}=\int_{\gamma_{j}} \Omega,
$$

where the $\gamma_{j}$ are basis cycles for the homology group. Mirror symmetry allows one to study the Yukawa field coupling constants as $(2,1)$-forms on the manifold $M$, by calculating the corresponding periods of $(1,1)$-forms on the mirror manifold $W$.

The idea of mirror symmetry in the theory of compactifications of superstrings has been intensively developed in the papers [1] - [11]. In particular, it has been effectively used in the study of periods on Calabi-Yau manifolds.

For a wide class of Calabi-Yau hypersurfaces (of arbitrary dimension $N-1$ ) in weighted projective space, the fundamental period of a holomorphic $(N-1)$-form $\Omega$ was computed in the paper [2]. Considered as a function on the moduli space, this period is a hypergeometric function, in fact representable as a Horn series (cf. Sect.1), which converges for large values of the distinguished modulus $\varphi_{0}$. Here arises the important problem to analytically continue the period into the region where the values of the modulus $\varphi_{0}$ are small, because such an analytic continuation allows one to calculate also the remaining periods of the form $\Omega$. By means of an 
artificial method for iterated summation of Horn series, the analytic continuation was carried out in [2], [3], [4] and [5], for some examples of hypersurfaces with two moduli.

In this paper we present a general method for analytic continuation of periods that depend on two or more moduli. Our approach consists in first representing the period as a multiple Mellin-Barnes integral (cf. Sect.3). The Mellin-Barnes integral is then in its turn calculated in terms of a series of multidimensional Grothendieck residues, and this latter computation is based on the method of separating cycles, which was developed by one of the authors in [13] and [14].

The idea of this method is based on the observation that the plane of integration may be viewed as the distinguished boundary (or edge) of many different polyhedra, and a choice of any such polyhedron that is also compatible with the form $\Omega$ produces its own residue formula for the Mellin-Barnes integral. (See [15] for the first realization of this idea in terms of a multidimensional Jordan residue lemma.) In this way, the different residue formulas provide an analytic continuation of the Mellin-Barnes integral (MB), and hence of the original period. Our study of the period may be illustrated by the following 3 -step scheme:

$$
\{\text { period }\} \rightarrow\{\text { Horn series }\} \rightarrow\{M B \text { integral }\} \rightarrow\{\text { new Horn series }\}
$$

In carrying out the second and third steps of the scheme we use the simple language of combinatorial geometry, related to the mutual positions of the polar hyperplanes of those gamma-functions that occur in the construction of the Horn series and of the Mellin-Barnes integrals.

\section{Periods on Calabi-Yau hypersurfaces in weighted projective spaces}

A weighted $N$-dimensional projective space is determined by a choice of $N+1$ natural numbers (weights) $k=\left(k_{0}, k_{1}, \ldots, k_{N}\right)$. Specifically, for non-zero vectors $x=\left(x_{0}, x_{1}, \ldots, x_{N}\right)$ in complex Euclidean space $\mathbb{C}^{N+1}$ one considers the equivalence relation

$$
x_{i} \sim \lambda^{k_{i}} x_{i}, \quad i=0,1, \ldots, N,
$$

where $\lambda$ is any non-zero complex number. It is the quotient space $\mathbb{C}^{N+1} / \sim$, with respect to this equivalence relation, that constitutes the weighted projective space of dimension $N$ and weight $k_{0}, k_{1}, \ldots, k_{N}$, and we denote it by $\mathbb{P}_{\left(k_{0}, k_{1}, \ldots, k_{N}\right)}^{N}=\mathbb{P}_{k}^{N}$. A hypersurface in weighted projective space $\mathbb{P}_{k}^{N}$ is defined to be the zero set

$$
M=\left\{x ; P_{0}(x)=0\right\}
$$

of a weighted-homogeneous polynomial (or superpotential) $P_{0}(x)$ of weight $k$. This means that

$$
P_{0}(x)=\sum_{\alpha \in A} c_{\alpha} x^{\alpha}=\sum_{\alpha_{0}, \ldots, \alpha_{N}} c_{\alpha_{0}, \ldots, \alpha_{N}} x_{0}^{\alpha_{0}} \cdots x_{N}^{\alpha_{N}},
$$

with every monomial $c_{\alpha} x^{\alpha}$ having the same weighted degree $d=k_{0} \alpha_{0}+\cdots+k_{N} \alpha_{N}$. We shall be interested in smooth hypersurfaces, and these are characterized the condition that the polynomial $P_{0}$ and its gradient $d P_{0}$ should have no common zeros in $\mathbb{C}^{N+1} \backslash\{0\}$. Moreover, we are going to assume the condition

$$
d=\sum_{i=0}^{N} k_{i}
$$


to hold, that is, the degree of the hypersurface should coincide with the sum of the components of the weight $k$. Condition (1.2) guarantees that the hypersurface (1.1) is a Calabi-Yau manifold: The first Chern class of $M$ is trivial, and $M$ admits no non-trivial holomorphic differential forms of degree $p=1,2, \ldots, N-2$. The next step in superstring theory is to aconsider a deformation of the manifold $M$ by means of a family of polynomials

$$
P_{\varphi}(x)=P_{0}(x)-\varphi_{0} x_{0} x_{1} \ldots x_{N}+\sum_{\beta \in B} \varphi_{\beta} x^{\beta} .
$$

Here the standard monomial $x_{0} x_{1} \cdots x_{N}$ and the monomials $x^{\beta}, \beta \in B$, that are added to $P_{0}(x)$, are chosen in such a way that their coefficients $\varphi_{0}$ and $\left\{\varphi_{\beta}\right\}$ provide a parametrization of the complex structures of the manifold $M$. For this reason these coefficients are called moduli, and the vector space $\varphi=\left\{\varphi_{0},\left\{\varphi_{\beta}\right\}\right\}$ is called the moduli space. In the description of the indicated complex structures, a crucial role is played by the holomorphic differential $(N-1)$-form

$$
\Omega=\Omega(\varphi)=\operatorname{Res}_{M_{\varphi}}\left[\frac{\mu}{P_{\varphi}(x)}\right]
$$

where the residue Res is taken with respect to the deformed submanifold

$$
M_{\varphi}=\left\{x ; P_{\varphi}(x)=0\right\}
$$

along which the meromorphic form $\mu / P_{\varphi}(x)$ has a pole [12]. In order that the quotient $\mu / P_{\varphi}(x)$, which is written in homogeneous coordinates $x=\left(x_{0}, x_{1}, \ldots, x_{N}\right) \in$ $\mathbb{C}^{N+1}$, should be a well defined differential form on the weighted projective space $\mathbb{P}_{k}^{N}$, in particular invariant with respect to the equivalence $x_{i} \sim \lambda^{k_{i}} x_{i}$, it is necessary that $\mu$ be of the type

$$
\mu=\sum_{i=0}^{N}(-1)^{i+1} k_{i} x_{i} d x_{0} \ldots[i] \ldots d x_{N}
$$

where $[i]$ means that the index $i$ is deleted. Since the form $\Omega$ is defined as the residue of a meromorphic form, it follows from the Leray residue formula ([12], Sect.21) that each period of $\Omega$ is given by some integral of the $N$-form $\mu / P_{\varphi}(x)$ over a $N$-dimensional cycle in $\mathbb{P}_{k}^{N} \backslash M_{\varphi}$. Any such integral may in its turn be reduced to a $(N+1)$-dimensional integral of the rational form $d x / P_{\varphi}(x)$, with $d x=d x_{0} d x_{1} \ldots d x_{N}$, in the affine space $\mathbb{C}^{N+1}$, (cf. [13], Sect. 20.1). In [16] it was proved that, if the denominator of a rational form $d x / Q(x)$ has fixed coefficients, then each vertex of the Newton polytope of $Q$, which does not lie on any of the coordinate planes, determines a residue of the rational form, and also that the collection of such residues is linearly independent. In our case some of the monomials in the denominator $P_{\varphi}(x)$ have variable coefficients $\varphi_{\alpha}$, and in this case one can associate a residue to every monomial $\varphi_{\beta} x^{\beta}$, for which $\beta$ is not in a coordinate plane, by taking the chosen coefficient $\varphi_{\beta}$ to be much bigger in absolute value than any other of the coefficients. Among the monomials with variable coefficients (which contribute to the deformation of the polynomial $P_{0}$ ) we have singled out the monomial $\varphi_{0} x_{0} \ldots x_{N}$, which in some sense corresponds to the center of the 
Newton polytope for $P_{0}$. It turns out that the residue associated to this monomial may be computed by developing the integrand in a Laurent series, and one calls this residue a fundamental period for the form $\Omega$. Its precise definition is as follows $[2]:$

$$
\omega_{0}(\varphi)=-\frac{\varphi_{0}}{(2 \pi i)^{N+1}} \int_{\gamma} \frac{d x_{0} \cdots d x_{N}}{P_{\varphi}(x)},\left|\varphi_{o}\right| \gg 1,
$$

the contour $\gamma$ being given as the Cartesian product of the $N+1$ circles $\left|x_{i}\right|=1$, $i=0,1, \ldots, N$. The coefficient in front of the integral is chosen so as to simplify notation in the resulting formulas.

Let us label by $c_{i} x^{\alpha^{i}}$ the monomials in the fixed part $P_{0}$ of the polynomial $P_{\varphi}$, and by $\varphi_{j} x^{\beta^{j}}$ the monomials in the variable part (except for $\varphi_{0} x_{0} \ldots x_{N}$ ). A computation (cf. formula (2.21) in [2], where $c_{i}=1$ ) now yields

$$
\varpi_{0}(\varphi)=\sum_{\left(n_{i}, m_{j}, r\right) \in L} \frac{\Gamma(r+1)}{\prod_{i} \Gamma\left(n_{i}+1\right) \prod_{j} \Gamma\left(m_{j}+1\right)} \frac{\prod_{i} c_{i}^{n_{i}} \prod_{j} \varphi_{j}^{m_{j}}}{\varphi_{0}^{r}},
$$

where the summation is performed over the sublattice $L$, of the integer lattice in the variables $n_{i}, m_{j}, r$, defined by the following system of $N+2$ linear homogeneous equations:

$$
\begin{gathered}
\sum_{i} \alpha_{k}^{i} n_{i}+\sum_{j} \beta_{k}^{j} m_{j}-r=0, \quad k=0, \ldots, N, \\
\sum_{i} n_{i}+\sum_{j} m_{j}-r=0 .
\end{gathered}
$$

Here $\alpha_{k}^{i}$ and $\beta_{k}^{j}$ are the coordinates of the vectors $\alpha^{i}, \beta^{j}$ in the lattice $\mathbb{Z}^{N+1}$. In the theory of multidimensional hypergeometric functions Laurent series such as (1.5) are called $\Gamma$-series (cf. [17]). There exists a standard procedure to go from $\Gamma$-series to Horn series by chosing a basis for the sublattice $L$. A Horn series is the same as a power series with coefficients of a special kind: They are quotients of products of gamma-functions composed with linear functions of the summation variables. In most models of Calabi-Yau manifolds the number of monomials in the polynomial $P_{0}$ is equal to $N+1$, the number of variables. It follows that in these cases one can incorporate the coefficients $c_{i}$ into the variables, that is, one may assume $c_{i}=1$. The choice of a basis for the sublattice $L$ transforms the series (1.5) into a power series of Horn type

$$
\varpi_{0}(t)=\sum_{m \in \mathbb{Z}_{+}^{n}} \frac{\Gamma(\langle a, m\rangle+1)}{\prod_{k=1}^{q} \Gamma\left(\left\langle a^{k}, m\right\rangle+1\right)} t_{1}^{m_{1}} \cdots t_{n}^{m_{n}},
$$

where $n$ is the dimension of the moduli space (i.e. the number of coefficients $\left.\left\{\varphi_{0}, \varphi_{j}\right\}\right)$, the vectors $a, a^{k} \in \mathbb{R}^{n}$ are determined from the monomial exponents $\alpha^{i}, \beta^{j}$, and the variables $t_{i}$ are monomials in the moduli $\varphi_{0}, \varphi_{j}$. Furthermore, the relation (weight balance)

$$
a=\sum_{k=1}^{q} a^{k}
$$


is satisfied.

As an illustration of what has been said so far, and also to illustrate results further on in the paper, we consider the following example, borrowed from [2]. By means of the polynomial

$$
P_{0}=x_{0}^{7}+x_{1}^{7} x_{3}+x_{3}^{3}+x_{2}^{7} x_{4}+x_{4}^{3}
$$

we obtain a Calabi-Yau hypersurface of degree 21 in the weighted projective space $\mathbb{P}_{(3,2,2,7,7)}^{4}$. With this hypersurface there is associated a 2-parameter family of manifolds

$$
M_{\varphi}=\left\{x_{0}^{7}+x_{1}^{7} x_{3}+x_{3}^{3}+x_{2}^{7} x_{4}+x_{4}^{3}-\varphi_{0} x_{0} x_{1} x_{2} x_{3} x_{4}+\varphi_{1} x_{0} x_{1}^{3} x_{2}^{6}=0\right\},
$$

which characterizes the complex structures on the hypersurface $P_{0}=0$. The fundamental period of the surface can be represented by a Horn series (cf. [2], formula $(4.19))$

$$
\sum_{m_{1}, m_{2} \geq 0} \frac{\Gamma\left(7 m_{1}+3 m_{2}+1\right)}{\Gamma^{2}\left(m_{1}+1\right) \Gamma^{2}\left(2 m_{1}+m_{2}+1\right) m_{1} ! m_{2} !} t_{1}^{m_{1}} t_{2}^{m_{2}}
$$

where $t_{1}=\varphi_{0}^{-7}, t_{2}=\varphi_{1} / \varphi_{0}^{3}$, while the factorials $m_{i} !=\Gamma\left(m_{i}+1\right)$ have been put aside to facilitate later computations.

\section{Mellin-Barnes integrals and their representations as Horn series}

By a $n$-dimensional Mellin-Barnes integral we mean an integral such as

$$
\Phi(t)=\frac{1}{(2 \pi i)^{n}} \int_{\gamma+i \mathbb{R}^{n}} \frac{\prod_{j=1}^{p} \Gamma\left(\left\langle a^{j}, z\right\rangle+b_{j}\right)}{\prod_{k=1}^{q} \Gamma\left(\left\langle c^{k}, z\right\rangle+d_{k}\right)}\left(-t_{1}\right)^{-z_{1}} \cdots\left(-t_{n}\right)^{-z_{n}} d z,
$$

where $a^{j}, c^{k}$ are vectors in $\mathbb{R}^{n}$, the numbers $b_{j}, d_{k}$ are also real, and the vector $\gamma \in \mathbb{R}^{n}$ is chosen so that the real $n$-dimensional set of integration

$$
\gamma+i \mathbb{R}^{n}=\left\{z \in \mathbb{C}^{n} ; z_{1}=\gamma_{1}+i y_{1}, \ldots, z_{n}=\gamma_{n}+i y_{n}\right\}
$$

does not intersect the polar hyperplanes

$$
\left\langle a^{j}, z\right\rangle+b_{j}=-\nu, \quad \nu=0,1,2, \ldots ; j=1, \ldots, p ;
$$

finally, $d z=d z_{1} \cdots d z_{n}$. In the one-variable case the integral (2.1) reduces to the following:

$$
\frac{1}{2 \pi i} \int_{\gamma-i \infty}^{\gamma+i \infty} \frac{\prod_{j} \Gamma\left(a_{j} z+b_{j}\right)}{\prod_{k} \Gamma\left(c_{k} z+d_{k}\right)}(-t)^{-z} d z
$$

The integral (2.2) was studied by Barnes, and it represents the inverse Mellin transform of the integrand. The behaviour of the integrals (2.1) is to a large extent governed by the following two quantities. The first one is the vector

$$
\Delta=\sum_{j} a^{j}-\sum_{k} c^{k}
$$


and the second one is the scalar quantity

$$
\alpha=\min _{\|y\|=1}\left(\sum_{j}\left|\left\langle a^{j}, y\right\rangle\right|-\sum_{k}\left|\left\langle c^{k}, y\right\rangle\right|\right),
$$

where $\|y\|=\left(y_{1}^{2}+\cdots+y_{n}^{2}\right)^{1 / 2}$ denotes the Euclidean norm in $\mathbb{R}^{n}$. In the case $n=1$ we have

$$
\alpha=\sum_{j}\left|a_{j}\right|-\sum_{k}\left|c_{k}\right|
$$

The number $\alpha$ determines the values of the complex parameters $t=\left(t_{1}, \ldots, t_{n}\right)$, for which the integral (2.1) converges, more precisely, it converges in the domain (cf. $[15])$

$$
U=\left\{t \in(\mathbb{C} \backslash 0)^{n} ;\left[\left(\pi-\arg t_{1}\right)^{2}+\cdots+\left(\pi-\arg t_{1}\right)^{2}\right]^{1 / 2}<\pi \alpha / 2\right\},
$$

which is non-empty for $\alpha>0$. The quantity $\Delta$ characterizes the domain in the space of integration variables $z$, in which the integrand is a decreasing function. The asymptotic Stirling formula shows namely that, outside the polar set, the expression under the integral sign in (2.1) decreases exponentially at infinity in any proper polyhedral cone in the halfspace

$$
\Pi_{\Delta, \gamma}=\left\{z \in \mathbb{C}^{n} ; \operatorname{Re}\langle\Delta, z\rangle<\langle\Delta, \gamma\rangle\right\} .
$$

The vector $\Delta$ being real, this halfspace may be viewed as the direct sum $\pi+i \mathbb{R}^{n}$ of the halfspace $\pi=\left\{x \in \mathbb{R}^{n} ;\langle\Delta, x\rangle<\langle\Delta, \gamma\rangle\right\}$ in the real subspace $\mathbb{R}^{n} \subset \mathbb{C}^{n}$, and the imaginary subspace $i \mathbb{R}^{n}$. In the the case $n=1$ the halfspace $\Pi_{\Delta, \gamma}$ is just the left or right halfplane (depending on the sign of $\Delta$ ), and $\pi$ is the left or right halfline on the real axis. Hence the quantity $\Delta$ plays a role similar to that of $\lambda$ in a Fourier type integral

$$
\int_{-\infty}^{+\infty} f(x) e^{i \lambda x} d x, \quad \lambda \in \mathbb{R}
$$

which for suitable functions $f(x)$ may be computed by means of the well known Jordan lemma ([18], Chap.5, Sect.2), as the sum of residues in the upper or lower halfplane, depending on the sign of $\lambda$. Now in the case where $\lambda=0$ this residue computation may be performed in either of the two halfplanes. We recall that the idea of the Jordan lemma consists in the following: The integral (2.5) is represented as the limit when $R \rightarrow \infty$ of the integrals $\int_{-R}^{+R}$; the interval of integration $[-R, R]$ is completed to a closed path by adding a halfcircle in the upper or lower halfplane; to the obtained closed contour one then applies the Cauchy residue theorem. Concerning the one-variable integrals (2.2), this idea was carried out by Barnes and others (cf. [19]), by replacing the upper and lower halfplanes by the left and right ones. And here it was the integrals (2.2) with zero characteristic $\Delta$ that attracted the most interest, because then they represent all the generalized hypergeometric functions. For $\Delta=0$ the integral (2.2) as a sum of residues in the left halfplane, 
as well as in the right one. It then turns out that the sum of residues in the left halfplane is a Horn power series in the variable $t$, convergent in some disk $|t|<r$, whereas the residue sum in the right halfplane represent another Horn series in the variable $t^{-1}$, which converges outside the same disk; and if the quantity $\alpha$ is positive (cf. (2.4a)), then each of these series is an analytic continuation of the other (cf. [19]).

In the multidimensional case the situation is somewhat more complicated and more varied. This is because for $n=1$ the vertical axis of integration $\operatorname{Re} z=\gamma$ is the boundary and also the edge of precisely two polyhedral cones, namely the left and right halfplanes. By a polyhedral cone we understand a set of the form

$$
\Pi=\left\{z \in \mathbb{C}^{n} ; \operatorname{Re} g_{j}(z)<r_{j}, \quad j=1, \ldots, m\right\},
$$

where $g_{j}(z)$ are linear functions with real coefficients. We shall be interested in simplicial cones, which correspond to $m=n$ with linearly independent functions $g_{j}, j=1, \ldots, n$ (such cones have $n$ faces of maximal dimension). The coefficients being real, one can write a polyhedral simplicial cone as $\pi+i \mathbb{R}^{n}$, where $\pi$ is an octant in the real subspace $\mathbb{R}^{n} \subset \mathbb{C}^{n}$ :

$$
\pi=\left\{x \in \mathbb{R}^{n} ; g_{j}(x)<r_{j}, j=1, \ldots, n\right\} .
$$

It follows that if $\gamma \in \mathbb{R}^{n}$ is the vertex of the octant $\pi$ (cf. Fig. 1), then the "vertical" set of integration $\gamma+i \mathbb{R}^{n}$ in the integral (2.1) is the edge of the cone $\Pi$ (cf. Fig. 2).

Since there is an infinite number of possible octants that all have $\gamma$ as their vertex, it follows that the "vertical" set of inegration is also the edge of infinitely many polyhedral cones. For the integral (2.1) one can accordingly obtain different residue formulas in cones with edge $\gamma+i \mathbb{R}^{n}$. Here we refer to local residues at the points of intersection of polar hyperplanes

$$
L_{j}^{\nu}=\left\{\left\langle a^{j}, z\right\rangle+b_{j}=-\nu\right\}, \quad \nu=0,1, \ldots, \quad j=1, \ldots, p .
$$

At an intersection point

$$
z=z_{J}^{m}=\underset{j_{1}}{m_{1}} \cap \cdots \cap L_{j_{n}}^{m_{n}},
$$


where precisely $n$ hyperplanes such as (2.6) come together, one may define a local Grothendieck residue

$$
\underset{z_{J}^{m}}{\operatorname{res}} \omega=\frac{1}{(2 \pi i)^{n}} \int_{\gamma_{J}^{m}} \omega,
$$

where $\omega$ is the integrand from (2.1), and $\gamma_{J}^{m}$ is a $n$-dimensional cycle

$$
\gamma_{J}^{m}=\left\{z ;\left|\left\langle a^{j_{1}}, z\right\rangle+b_{j_{1}}+m\right|=\cdots=\left|\left\langle a^{j_{n}}, z\right\rangle+b_{j_{n}}+m_{n}\right|=\varepsilon\right\},
$$

with $\varepsilon>0$ sufficiently small. By means of the coordinate change

$$
\mathrm{w}_{j}=\left\langle a^{j}, z\right\rangle+b_{j}+m_{j}, \quad j=1, \ldots, n,
$$

and invoking the Cauchy formula, it is easily verified that

$$
\underset{z_{J}^{m}}{\operatorname{res}} \omega=\frac{(-1)^{|m|}}{m ! \Delta_{J}} \frac{\prod_{j \neq J} \Gamma\left(\left\langle a^{j}, z_{j}^{m}\right\rangle+b_{j}\right)}{\prod_{k=1}^{q} \Gamma\left(\left\langle c^{k}, z_{J}^{m}\right\rangle+d_{k}\right)}\left(-t_{1}\right)^{\left(z_{J}^{m}\right)_{1}} \cdots\left(-t_{n}\right)^{\left(z_{J}^{m}\right)_{n}},
$$

where $\Delta_{J}=\operatorname{det}\left(a^{j_{1}}, \ldots, a^{j_{n}}\right),\left(z_{J}^{m}\right)_{i}$ are the coordinates of the point $z_{J}^{m}$, and $|m|=m_{1}+\cdots+m_{n}, m !=m_{1} ! \cdots m_{n} !$

If there are more than $n$ complex hyperplanes that meet at the point $z$, then this set of hyperplanes is divided into $n$ groups ( $n$ divisors) $D_{1}, \ldots, D_{n}$, and one defines

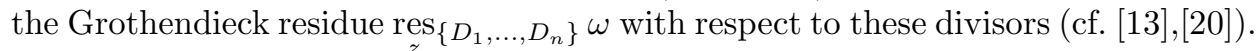
In this case the residue formula becomes more complicated, and along with powers of the variables $t_{j}$ it will also involve powers of logarithms $\left(\ln t_{1}\right)^{p_{1}} \cdots\left(\ln t_{n}\right)^{p_{n}}$, with $p_{1}+\cdots+p_{n}$ at least $n$ units less than the number of hyperplanes that intersect at the given point. Now we notice that the expression (2.7) corresponds to a term in a Horn series (its coefficient is a quotient of products of gamma-functions with arguments that are linear in $\left.m=\left(m_{1}, \ldots, m_{n}\right)\right)$. In the terminology of hypergeometric function theory [17], the formula (2.7) (i.e. the residue expression, when there are just $n$ intersecting polar planes) corresponds to the non-resonance case.

Next we observe that the solution $z_{J}^{m}$ to the system of linear equations

$$
\left\langle a^{j_{1}}, z\right\rangle+b_{j_{1}}=-m_{1}, \ldots\left\langle a^{j_{n}}, z\right\rangle+b_{j_{n}}=-m_{n}
$$

can be linearly parametrized also with respect to $m=\left(m_{1}, \ldots, m_{n}\right)$ as follows:

$$
\left(z_{J}^{m}\right)_{1}=\left\langle A^{j_{1}}, m\right\rangle+B_{j_{1}}, \ldots\left(z_{J}^{m}\right)_{n}=\left\langle A^{j_{n}}, m\right\rangle+B_{j_{n}} .
$$

Hence, factoring out the monomial $(-t)^{B_{J}}=\left(-t_{1}\right)^{B_{j_{1}}} \cdots\left(-t_{n}\right)^{B_{j_{n}}}$ in $(2.7)$ and making the substitution $\tau_{i}=t_{1}^{a_{i}^{j_{1}}} \ldots t_{n}^{a_{i}^{j_{n}}}, i=1, \ldots, n$, the expression (2.7) becomes a term of a Horn power series in the variables $\tau_{1}, \ldots, \tau_{n}$.

Now we turn to the question of deciding through which residues (2.7) one should express the integral (2.1). The case $n=2, \Delta \neq 0$ was considered in [15]. In this case, just like in one variable, the integral (2.1) is represented by only one residue formula:

$$
\Phi(t)=\sum_{z_{J}^{m} \in \Pi_{\Delta, \gamma}} \underset{z_{J}^{m}}{\operatorname{res}} \omega
$$


where the summation is over all intersection points $z_{J}^{m}=L_{j_{1}}^{m_{1}} \cap L_{j_{2}}^{m_{2}}$ in the halfspace $\Pi_{\Delta, \gamma}$, for which the lines $L_{j_{1}}^{m_{1}}$ and $L_{j_{2}}^{m_{2}}$ intersect the line

$$
l=\left\{x \in \mathbb{R}^{2} ;\langle\Delta, x\rangle=\langle\Delta, \gamma\rangle\right\}
$$

on different sides of the point $\gamma \in l$ (for this reason the cycle of integration $\gamma+i \mathbb{R}^{2}$ is said to be a separating cycle for the complex lines $\left.L_{j_{1}}^{m_{1}}, L_{j_{2}}^{m_{2}}\right)$.

Turning to the case of interest to us $\Delta=0$, we introduce the following concept. In the plane $\mathbb{R}^{2}$ we consider an arbitrary angle (cone) $\pi$ with vertex $\gamma$ (cf. Fig. 1 ). We shall say that the angle $\pi$ is compatible with the family (2.7) of polar lines $L_{j}^{\nu}$ of the integrand (2.1), if each such line intersects at most one side of the angle $\pi$ (in Fig.3 the angles $\pi_{1}, \pi_{2}, \pi_{3}$ are compatible with the family consisting of vertical, horizontal and oblique lines). Then in accordance with the multi-dimensional Jordan lemma [15], we have the following

Claim 1. Let $\Delta=0$. If the angle $\pi$ is compatible with the family $L_{J}^{\nu}=\left\{\left\langle a^{j}, z\right\rangle+\right.$ $\left.b_{j}=-\nu\right\}$ of polar lines for the integral (2.1), then this integral is given by the residue formula

$$
\Phi(t)=\sum_{z_{J}^{m} \in \pi}^{\underset{z_{J}^{m}}{\operatorname{res}} \omega}
$$

where the summation is performed over all intersection points $z_{J}^{m}=L_{j_{1}}^{m_{1}} \cap L_{j_{2}}^{m_{2}}$ in $\pi$, for which $L_{j_{1}}^{m_{1}}$ intersects only one side of $\pi$, while $L_{j_{2}}^{m_{2}}$ only meets the other side. Furthermore, the series converges in a domain of the form

$$
\left\{t \in \mathbb{C}^{2} ;\left|t_{1}^{A_{1}} t_{2}^{B_{2}}\right|<r_{1}, \ldots,\left|t_{1}^{A_{s}} t_{2}^{B_{s}}\right|<r_{s}\right\} .
$$

In sections 3 and 4 we will present the applications of the multidimensional $(n>2)$ Jordan lemma that are of primary interest to us, but here we consider the following two-dimensional example:

$$
\Phi(t)=\frac{1}{(2 \pi i)^{2}} \int_{\gamma+i \mathbb{R}^{2}} \frac{\Gamma\left(z_{1}\right) \Gamma\left(z_{2}\right) \Gamma\left(-7 z_{1}-3 z_{2}+1\right)}{\Gamma^{2}\left(-z_{1}+1\right) \Gamma^{2}\left(-2 z_{1}-z_{2}+1\right)}\left(-t_{1}\right)^{z_{1}}\left(-t_{2}\right)^{z_{2}} d z_{1} d z_{2}
$$


¿From the results in section 3 it will follow that this integral represents the series (1.9), and hence the fundamental period of the manifold (1.8). Here we have three families of polar lines

$$
L_{1}^{\nu}=\left\{z_{1}=-\nu\right\}, L_{2}^{\nu}=\left\{z_{2}=-\nu\right\}, L_{3}^{\nu}=\left\{-7 z_{1}-3 z_{2}+1=-\nu\right\},
$$

as indicated in Fig.3. In the cone $\pi_{1}$ it is only lines from the first two families that intersect, i.e. poles of the gamma-functions $\Gamma\left(z_{1}\right)$ and $\Gamma\left(z_{2}\right)$. The intersection points $L_{1}^{m_{1}} \cap L_{2}^{m_{2}}$ can be parametrized as $\left(z_{1}, z_{2}\right)=\left(-m_{1},-m_{2}\right)$, and therefore, by Claim 1 and formula (2.7), the integral $\Phi(t)$ is given by a Horn series (1.9).

In this example $\pi_{1}$ is not the only angle that is compatible with the polar family $L_{j}^{\nu}$. Therefore there exist other residue formulas for $\Phi(t)$, which also provide analytic continuation of the series (1.9). Now, in $\pi_{2}$ it is the poles of the functions $\Gamma\left(z_{1}\right)$ and $\Gamma\left(-7 z_{1}-3 z_{2}+1\right)$ that intersect. These points of intersection admit the parametrization

$$
\left(z_{1}, z_{2}\right)=\left(-m_{2}, \frac{1}{3} m_{1}+\frac{7}{3} m_{2}+\frac{1}{3}\right) .
$$

The determinant $\Delta_{31}$ equals 3 , and hence it follows from Claim 1 and formula (2.7) that the integral (2.8) may be represented by the series

$$
\frac{\left(-t_{2}\right)^{-\frac{1}{3}}}{3} \sum_{m_{1}, m_{2} \geqslant 0} \frac{\Gamma\left(\frac{1}{3} m_{1}+\frac{7}{3} m_{2}+1\right) e^{\pi i\left(\frac{2}{3} m_{1}-\frac{7}{3} m_{2}\right)}\left(\frac{1}{t_{2}^{1 / 3}}\right)^{m_{1}}\left(\frac{t_{1}}{t_{2}^{7 / 3}}\right)^{m_{2}}}{m_{1} ! m_{2} ! \Gamma^{2}\left(m_{2}+1\right) \Gamma^{2}\left(-\frac{1}{3} m_{1}-\frac{1}{3} m_{2}+\frac{2}{3}\right)},
$$

which is convergent in a domain in the variables $t_{1}, t_{2}$, which is different from the one for the series (1.9).

Finally, in the angle $\pi_{3}$ it is the poles of the functions $\Gamma\left(z_{2}\right)$ and $\Gamma\left(-7 z_{1}-3 z_{2}+1\right)$ that meet. The intersection point are given by

$$
\left(z_{1}, z_{2}\right)=\left(\frac{3}{7} m_{1}+\frac{1}{7} m_{2}+\frac{1}{7},-m_{1}\right) .
$$

The determinant $\Delta_{23}$ is equal to 7 , and so this time we obtain the representation

$$
\frac{\left(-t_{1}\right)^{-\frac{1}{7}}}{7} \sum_{m_{1}, m_{2} \geqslant 0} \frac{\Gamma\left(\frac{3}{7} m_{1}+\frac{1}{7} m_{2}+\frac{1}{7}\right) e^{\pi i\left(\frac{6}{7} m_{2}-\frac{3}{7} m_{1}\right)}\left(\frac{t_{2}}{t_{1}^{3 / 7}}\right)^{m_{1}}\left(\frac{1}{t_{1}^{1 / 7}}\right)^{m_{2}}}{m_{1} ! m_{2} ! \Gamma^{2}\left(-\frac{3}{7} m_{1}-\frac{1}{7} m_{2}+\frac{6}{7}\right) \Gamma^{2}\left(\frac{1}{7} m_{1}-\frac{2}{7} m_{2}+\frac{5}{7}\right)}
$$

The fact that the series (2.9) and (2.10) are analytic continuations of the series (1.9) is a consequence of a result that we provide in section 4 .

\section{Representation of periods by Mellin-Barnes integrals}

We know that the fundamental period $\varpi$ can be expressed as a Horn series (1.6). We shall now represent this series in the form of a Mellin-Barnes integral (2.1).

Claim 2. If in the series (1.6) the vector a have positive components, then this series admits a representation as the Mellin-Barnes integral

$$
\varpi_{0}(t)=\frac{1}{(2 \pi i)^{n}} \int_{\gamma+i \mathbb{R}^{n}} \frac{\prod_{j=1}^{n} \Gamma\left(z_{j}\right) \Gamma\left(1-z_{j}\right) \Gamma(1-\langle a, z\rangle)}{\prod_{k=1}^{q} \Gamma\left(1-\left\langle a^{k}, z\right\rangle\right)}(-t)^{-z} d z
$$


where $\gamma$ is any point in the polytope

$$
\Pi=\left\{x \in \mathbb{R}^{n} ; 0<x_{j}<1, j=1, \ldots, n,\langle a, x\rangle<1\right\},
$$

and $(-t)^{z}=\left(-t_{1}\right)^{-z_{1}} \cdots\left(-t_{n}\right)^{-z_{n}}$.

To prove this claim we consider the polyhedron $\Pi=\pi+i \mathbb{R}^{n}$, where

$$
\pi=\left\{x \in \mathbb{R}^{n} ; x_{j} \leqslant \gamma_{j}, j=1, \ldots, n\right\},
$$

with $\gamma_{j}$ being the components of the vector $\gamma$. The integrand in (3.1) has $2 n+1$ families of polar hyperplanes $\left\{z_{j}=-\nu\right\},\left\{1-z_{j}=-\nu\right\}$, and $\{1-\langle a, z\rangle=-\nu\}$, of which only the first $n$ families intersect the polyhedron $\Pi$. Each of these families form a divisor

$$
D_{j}=\bigcup_{\nu=0}^{\infty}\left\{z_{j}=-\nu\right\}, \quad j=1, \ldots, n .
$$

The collection of divisors $D_{j}$ is compatible with the polyhedron $\Pi$ (cf. [15]) in the sense that each $D_{j}$ is disjoint from the corresponding face of $\Pi$. More precisely, if $\pi_{j}=\left\{x \in \pi ; x_{j}=\gamma_{j}\right\}$ is a face of the octant $\pi$, then $\Pi_{j}=\pi_{j}+i \mathbb{R}^{n}$ is a face of the polyhedron $\Pi$, and the compatibility conditions

$$
D_{j} \cap \Pi_{j}=\varnothing, \quad j=1, \ldots, n
$$

are clearly satisfied. By the abstract multidimensional Jordan lemma [15] the integral (3.1) is equal to the sum of Grothendieck residues of the integrand in the polyhedron $\Pi$. Each residue is located at a point $\left(-m_{1}, \ldots,-m_{n}\right)$, and it is equal to

$$
\begin{aligned}
\frac{(-1)^{|m|}}{m !} \frac{\prod_{j=1}^{n} \Gamma\left(1+m_{j}\right) \Gamma(1+\langle a, m\rangle)}{\prod_{k=1}^{q} \Gamma\left(1+\left\langle a^{k}, m\right\rangle\right)}(-t)^{m} & \\
& =\frac{\Gamma(1+\langle a, m\rangle)}{\prod_{k=1}^{q} \Gamma\left(1+\left\langle a^{k}, m\right\rangle\right)} t_{1}^{m_{1}}, \ldots, t_{n}^{m_{n}} .
\end{aligned}
$$

This completes the proof of our claim.

\section{Analytic continuation of the periods}

We observe that we have already carried out the first two steps in our scheme for the study of the fundamental period, that we mentioned in the introduction of this paper. Indeed, in section 1 we went through the first step: $\{$ period $\} \Longrightarrow$ \{Horn series\} (formula (1.6)), and in section 3 the second step: \{Horn series\} $\Longrightarrow\{$ Mellin-Barnes integral $\}$ was accomplished (formula (3.1.)). The results of section 3 will now allow us to perform the third and final step in the scheme, i.e. to calculate the Mellin-Barnes integrals in various ways, thereby establishing the analytic continuation of the fundamental period.

Let us assume that in the series (1.6) the vector $a$ has positive components $a_{j}$, and that all factors $\Gamma\left(m_{j}+1\right)=m_{j} !, j=1, \ldots, n$, are present in the denominator of the coefficients. Let these be the last factors $\Gamma\left(m_{j}+1\right)=m_{j} !, j=1, \ldots, n$, 
where $s=q-n$. It is not hard to see that in this case the integral representation (3.1) of the series will have the appearence

$$
\varpi_{0}(t)=\frac{1}{(2 \pi i)^{n}} \int_{\gamma+i \mathbb{R}^{n}} \frac{\prod_{j=1}^{n} \Gamma\left(z_{j}\right) \Gamma(1-\langle a, z\rangle)}{\prod_{k=1}^{s} \Gamma\left(1-\left\langle a^{k}, z\right\rangle\right)}(-t)^{-z} d z,
$$

with the balance condition

$$
a-\sum_{k=1}^{s} a^{k}=(1, \ldots, 1)
$$

for the weights. Remark. The authors are not aware of any case of a series (1.6) representing a period on a Calabi-Yau hypersurface in weighted projective space, for which the positivity condition on the coordinate vector $a$ is not satisfied. However, in the paper [2] (formula (5.3)) an example of a period is given for which the factorials $m_{i}$ ! are missing in its series representation of type (1.6). It is conspicuous that the corresponding Calabi-Yau manifold lacks a mirror partner in weighted projective space [2], [8]. As shown in the paper [11], this missing mirror image may however be realized as a hypersurface in a more general toric manifold [9].

We now formulate our main result regarding the integral (4.1):

Claim 3. For each $j$ from 1 to $n$ the integral (4.1) admits the series representation

$\varpi_{j}(t)=\frac{(-1)^{j-1}}{a_{j}} \sum_{m \geq 0} \frac{(-1)^{|m|} \Gamma\left(\frac{1+\left\langle A^{j}, m\right\rangle}{a_{j}}\right)\left(-t_{1}\right)^{m_{1}} \cdots\left(-t_{j}\right)^{-\frac{1+\left\langle A^{j}, m\right\rangle}{a_{j}}} \cdots\left(-t_{n}\right)^{m_{n}}}{m ! \prod_{k=1}^{s} \Gamma\left(\left(a_{j}-a_{j}^{k}+\left\langle A^{k j}, m\right\rangle\right) / a_{j}\right)}$,

where $a_{j}, a_{j}^{k}$ are the components of the vectors $a$ and $a^{k}$, the vector $A^{j}$ equals $\left(a_{1}, \ldots, 1, \ldots, a_{n}\right)$ with 1 in the $j$ 'th slot, the vectors $A^{k j}$ are given by

$$
\left(a_{j} a_{1}^{k}-a_{1} a_{j}^{k}, \ldots,-a_{j}^{k}, \ldots, a_{j} a_{n}^{k}-a_{n} a_{j}^{k}\right)
$$

and as usual $|m|=m_{1}+\cdots+m_{n}$ and $m !=m_{1} ! \cdots m_{n}$ !. Moreover, if for the integral (4.1) the quantity $\alpha$, defined by formula (2.4), is positive, then each of the series $\varpi_{j}(t)$ provides an analytic continuation of the fundamental period $\varpi_{0}(t)$ to its corresponding domain of convergence.

For instance, for the integral (2.8) the quantity $\alpha$ is equal to $2 / \sqrt{58}$, and hence the series (2.9), (2.10) do give analytic continuations of the series (1.9).

To prove the formulated claim one may argue as in the proof of Claim 2. Indeed, for each $j=1, \ldots, n$ we consider the polyhedron $\Pi_{j}=\pi_{j}+i \mathbb{R}^{n}$, where $\pi_{j}$ is the octant

$$
\pi_{j}=\left\{x \in \mathbb{R}^{n} ; x_{i} \leq \gamma_{i}, i=1, \ldots[j] \ldots, n,\langle a, x\rangle>\langle a, \gamma\rangle\right\} .
$$

It is evident that $\gamma+i \mathbb{R}^{n}$ is an edge for this polyhedron, and that the polar divisor of the function $\Gamma\left(z_{j}\right)$ does not intersect $\Pi_{j}$. Furthermore, the divisors

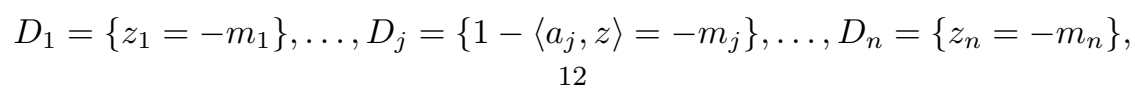


which represent the poles of the functions $\Gamma\left(z_{1}\right), \ldots, \Gamma\left(1-\left\langle a_{j}, z\right\rangle\right), \ldots, \Gamma\left(z_{n}\right)$, are compatible with the polyhedron $\Pi_{j}$. Hence, according to the multidimensional Jordan lemma the integral (4.1) is equal to the sum of Grothendieck residues with respect to the divisors $D_{1}, \ldots, D_{n}$ at their intersection points in $\Pi_{j}$. Applying the residue formula at these points, we find that the integral is equal to $\varpi_{j}(t)$ as claimed.

If the quantity $\alpha$, given in formula (2.4), is positive for the integral (4.1), then the domain of convergence for this integral will intersect each of the respective domains of convergence for the series $\varpi_{j}(t)$. Consequently, in this case these latter series analytically continue $\varpi_{0}(t)$ into their respective convergence domains.

In conclusion we remark that one-dimensional Mellin-Barnes integrals have been commonly used in mathematical physics [21] in connection with questions of asymptotics of determinants in string theory, high temperature limits for free energy of scalar fields in curved space-time etc. It is our belief that it would be adequate to study some of these questions in greater generality by means of multidimensional Mellin-Barnes integrals.

Acknowledgements Passare and Tsikh are indebted to the Royal Swedish Academy of Sciences for its financial support. Cheshel wishes to express his gratitude towards V. Rabotin and B. Rubtsov for useful discussions, and towards A. Niemi for his hospitality at the Department of Theoretical Physics of Uppsala University (Sweden).

\section{REFERENCES}

1. T.Hübsch, Calabi-Yau Manifolds - A Bestiary for Physicists, World Scientific, Singapore, 1992.

2. P.Berglund, P.Candelas, X. de la Ossa, A.Font, T.Hubsch, D.Janćić \& F.Quevedo, Nucl.Phys. B419 (1994), 352.

3. P.Candelas, X. de la Ossa, A.Font, S.Katz \& S.R.Morrison, Nucl.Phys. B416 (1994), 481.

4. P.Candelas, A.Font, S.Katz \& S.R.Morrison, Mirror Symmetry for Two Parameter Model2, preprint hep-th /9403187.

5. P.Berglund, E.Derrick, T.Hübsch \& D.Janćić, On Periods for String Compactifications, preprint HUPAPP-93/6, IASSNS-HEP-93/80, UTTG-27-93..

6. S.Hosono, A.Klemm, S.Taisen \& S.-T.Yau, Mirror Symmetry, Mirror Map and Application to Calabi-Yau Hupersurfaces, preprint hep-th /9308083.

7. P.Candelas, X. de la Ossa, P.Greene \& L.Parkes, Nucl. Phys. B359 (1991), 21.

8. B.R.Greene and M.R.Plesser, Nucl.Phys. B338 (1990).

9. V.V.Batyrev, Duke Math. Journ. 69 (1993), 349.

10. P.Candelas, X. de la Ossa \& S.Katz, Mirror Symmetry for Calabi-Yau Hupersurfaces in Weighted $\mathbb{C} P(4)$ and Extensions of Landau-Ginzburg Theory, hep-th /9412117.

11. P.Berglund \& S.Katz, Nucl. Phys. B420 (1994), 289, hep-th /9311014.

12. J.Leray, Le calcul différentiel et intégral sur une variété analytique complexe (Problème de Cauchy, III), Bull. Soc. math. France 87 (1959), 81-180.

13. A.Tsikh, Multidimensional Residues and Their Applications, Translations of Mathematical Monographs, vol. 103, AMS, Providence, 1992.

14. A.Tsikh, Methods in the Theory of Multidimensional Residues (Doctoral dissertation in Russian), Novosibirsk: Math. Inst., Russian Acad. of Sci. (Siberian branch), 1990.

15. M.Passare, A.Tsikh \& O.Zhdanov, A multidimensional Jordan residue lemma with an application to Mellin-Barnes integrals, Aspects of Math. E26 (1994), 233-241.

16. M.A.Mkrtchyan \& A.P.Yuzhakov, Newton polytopes and Laurent series for rational functions of $n$ variables (in Russian), Izv. Akad. Nauk Arm. SSR 17 (1992), 99-105.

17. I.M.Gel'fand, A.V.Zelevinsky \& M.M.Kapranov, Hypergeometric functions and toric manifolds, Funct. Anal. Appl. 28 (1989), 94. 
18. A.G.Sveshnikov \& A.N.Tikhonov, Theory of Functions of a Complex Variable (in Russian), "Nauka", Moscow, 1967.

19. O.I.Marichev, Methods for Computing Integrals of Special Functions, "Nauka", Minsk, 1978.

20. Ph.Griffiths \& J.Harris, Principles of Algebraic Geometry, "John Wiley \& Sons", New York, 1978.

21. E.Elizalde, K.Kirsten \& S.Zerbini, Applications of the Mellin-Barnes integral representation, hep-th /9501048. 


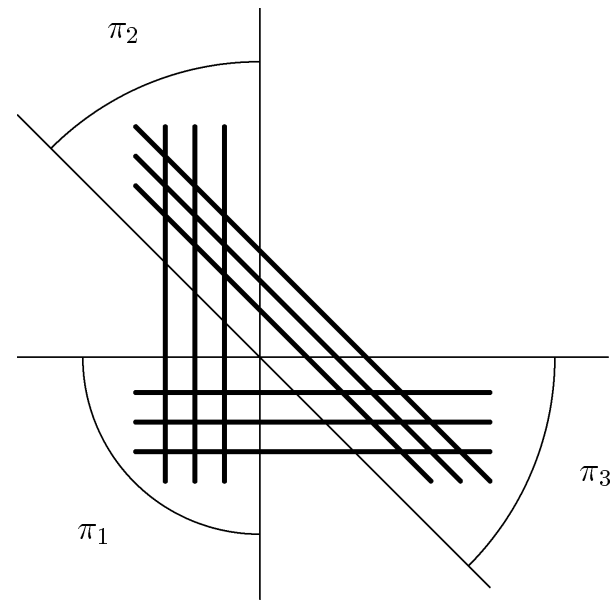

Fig. 3 


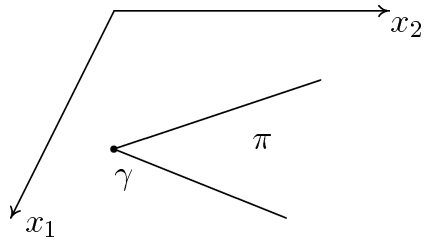

Fig. 1

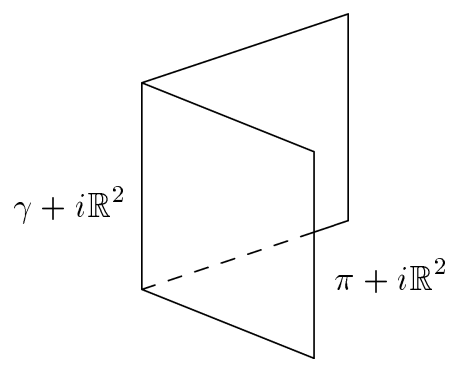

Fig. 2 